\title{
Directed Evolution of an Efficient and Thermostable PET Depolymerase
}

Elizabeth L. Bell', Ross Smithson, Siobhan Kilbride, Jake Fosterl, Florence Hardy, Saranarayanan Ramachandran ${ }^{2}$, Aleksander Tedstone, Sarah J. Haigh ${ }^{2}$, Arthur Garforth ${ }^{3}$, Philip J. R. Day ${ }^{4}$, Colin Levy', Michael P. Shaver ${ }^{2}$ E Anthony P. Green ${ }^{1^{*}}$

${ }^{1}$ Manchester Institute of Biotechnology, 131 Princess Street, University of Manchester, Manchester, MI 7DN, UK.

${ }^{2}$ Department of Materials, The University of Manchester, Manchester M13 9PL, UK.

${ }^{3}$ Department of Chemical Engineering and Analytical Science, University of Manchester, Oxford Road, Manchester, M1 3AL,UK.

${ }^{4}$ Division of Evolution and Genomic Sciences, Faculty of Biology, Medicine and Health, The University of Manchester, Manchester M13 9PL, UK.

*Email: anthony.green@manchester.ac.uk

\section{Abstract}

The recent discovery of a hydrolytic enzyme, IsPETase, that can deconstruct poly(ethylene) terephthalate (PET), has sparked great interest in biocatalytic approaches to recycle plastics. Realisation of commercial utility will require the development of robust engineered enzymes that meet the demands of industrial processes. Although rationally engineered variants of PETases have been reported, enzymes that have been experimentally optimised through iterative rounds of directed evolution - the go-to method for engineering industrially useful biocatalysts - have not yet been described. Here, we report the development and implementation of an automated, high-throughput directed evolution platform for engineering polymer degrading enzymes. Evaluation of >13,000 IsPETase variants, applying catalytic activity at elevated temperatures as a primary selection pressure, afforded a HotPETase variant with 21 mutations that has a melting temperature of $82.5^{\circ} \mathrm{C}$ and can therefore operate near or above the glass transition temperature of PET $\left(60-70^{\circ} \mathrm{C}\right)$. HotPETase can depolymerise semi-crystalline PET more rapidly than previously reported PETases and can selectively deconstruct the PET component of a laminated packaging multi-material. Structural characterisation of HotPETase reveals several interesting features that have emerged during evolution to improve thermotolerance and catalytic performance. Our study establishes laboratory evolution as a platform to engineer useful plastic degrading enzymes to underpin biocatalytic plastic recycling processes. 


\section{Introduction}

Fossil-fuel derived plastics have become an essential commodity in modern society. Their versatility, light weight and low production costs have led to an ever-growing appetite for these synthetic polymers, with an estimated 360 million tonnes of plastic being manufactured each year. ${ }^{1}$ Unfortunately, the properties that make plastics appealing in protecting products, food and medicines, such as their inertness and durability, mean that they become problematic and persistent pollutants if disposed of incorrectly. It is now widely recognized that the accumulation of plastics in the environment poses a serious global pollution threat, with marine ecosystems being particularly at risk. ${ }^{2}$ There is therefore urgent need for new technologies to circularize plastic waste, retaining value in these important materials whilst preventing an environmental catastrophe. ${ }^{3}$

Poly(ethylene terephthalate) (PET) is amongst the most abundantly produced synthetic polymers. Demand for this material has grown substantially due to a heightened global appetite for convenient single-use containers, ${ }^{4,5}$ with an estimated 1 million PET bottles being produced every minute. ${ }^{6}$ Although mechanical recycling methods are available for PET, recycling rates remain low due to difficulties in collecting and sorting mixed postconsumer waste streams, ${ }^{3,7}$ and declining polymer properties after repeated processing cycles. $^{8}$ In light of these challenges, depolymerisation via chemical recycling, including solvolysis methods such as hydrolysis and glycolysis, ${ }^{9,10}$ has garnered interest as a means of circularising the PET life-cycle. Unfortunately, chemical recycling methods are often energy intensive, can require large volumes of organic solvents and, for non-selective pyrolytic methods, can produce mixtures of products that are difficult to separate. ${ }^{\text {ll }}$ This is especially true for real-world products and packaging, where lamination of plastics creates multimaterials with unique end-of-life challenges.

In recent years, biological catalysis has emerged as a potentially attractive alternative to more traditional plastic recycling strategies. ${ }^{12}$ Enzymes are the most proficient and selective catalysts known and can accelerate chemical processes with unrivalled specificities. In principle, mixed waste streams could be selectively degraded by enzymes without need for manual sorting, ${ }^{13}$ and the resulting deconstruction products tailored and fed into additional enzymatic cascades to create higher value chemical feedstocks and products, ${ }^{14}$ all carried out under low cost, low carbon, environmentally benign reaction conditions. ${ }^{\text {Il }}$ For enzymatic PET recycling to be feasible, suitable biocatalysts must first be discovered and then 
engineered to tailor their properties for target applications. Unfortunately, while microorganisms are extremely well-equipped to deconstruct biological polymers such as proteins, DNA and carbohydrates, they are generally not well-adapted to achieve efficient depolymerisation of anthropogenic polymers. ${ }^{15}$ Nevertheless, some cutinases have been shown to have promiscuous PET degradation abilities. ${ }^{16-19}$ These enzymes typically display poor activity towards PET materials with characteristics commonly found in post-consumer waste. To function effectively, even engineered cutinases require the extensive preprocessing of PET substrates to amorphize the material, ${ }^{20}$ a process which compromises the physical properties of the PET, and also the economics and environmentally sustainability of biocatalytic plastic recycling approaches. ${ }^{13}$

The recent serendipitous discovery of an organism, Ideonella sakaiensis, with the ability to use PET as a carbon source, ${ }^{21}$ revealed a naturally-evolved, PET-hydrolysing enzyme $\left(\right.$ IsPETase $\left.{ }^{\mathrm{WT}}\right)$, that has an enhanced ability to depolymerise more crystalline forms of PET. $^{21,22}$ There are interesting structural differences between IsPETase and homologous cutinases, which are thought to be linked to this improved activity, ${ }^{22}$ including a conformationally flexible Trp185 that has been proposed to aid polymer binding. ${ }^{23,24}$ The unique catalytic properties of IsPETase make it an attractive candidate as a biocatalyst for PET recycling. Unfortunately, the wildtype enzyme suffers from low thermostability, ${ }^{21}$ meaning that biotransformations must be run at ambient temperatures far below the glass transition temperature $\left(T_{\mathrm{g}}\right)$ of $\mathrm{PET}\left(T_{\mathrm{g}} \sim 70^{\circ} \mathrm{C}\right)$, which compromises polymer deconstruction rates. $^{25,26}$

In an effort to address these limitations, improvements in PETase stability have been achieved using a variety of rational engineering approaches. ${ }^{22,27-30}$ In contrast, experimental optimisation of IsPETase using directed evolution, which typically offers a more comprehensive approach to enzyme engineering, ${ }^{31,32}$ remains under-explored, likely due to the lack of suitable protocols for monitoring the deconstruction of insoluble plastics with sufficient throughput. ${ }^{12}$ Here we establish an automated, high-throughput directed evolution platform for engineering plastic deconstructing enzymes and showcase its utility by engineering a thermostable variant of IsPETase which can operate at the glass transition temperature of PET. This engineered biocatalyst can efficiently depolymerise semicrystalline PET and can selectively deconstruct real-world laminated packaging materials. 


\section{Results}

We selected a rationally designed 'thermostable' variant of IsPETase containing three mutations, S121E, D186H, R280A (IsPETase ${ }^{\mathrm{TS}}, T_{\mathrm{m}}=56.8^{\circ} \mathrm{C}$ ), ${ }^{27}$ as a starting point for engineering. Consistent with previous reports, IsPETase ${ }^{\mathrm{TS}}$ displays superior PET degradation activity compared to the wild-type enzyme at $40^{\circ} \mathrm{C}$, however, this activity decreases dramatically at more elevated temperatures (Extended Data, Fig. 1).

Our first objective was to develop a high-throughput screening workflow to underpin the directed evolution study. To this end, individual enzyme variants were evaluated as crude cell lysates arrayed in 96-well plates, using amorphous PET film (amoPET, 0 \% crystallinity, sourced from Goodfellow) as the reaction substrate. The amoPET substrate allowed us to prepare uniform $6 \mathrm{~mm}$ diameter discs that could be easily placed into individual wells to allow fair comparison of variant activity (Fig. 1). Interestingly, we found that the addition of our standard chemical cell lysis reagents, lysozyme and polymyxin B, resulted in the production of a lysate with substantially reduced activity (Extended Data, Fig. 2), hence, we developed a method for 96-well plate cell lysis using the commercial reagent, BugBuster. The accumulation of mono(2-hydroxyethyl) terephthalate (MHET) and terephthalic acid (TPA) in the reaction supernatant was monitored by Ultra Performance Liquid Chromatography (UPLC); these two products result from the partial and complete hydrolysis of the PET backbone, respectively, and are the known major products of IsPETase-mediated depolymerizations. ${ }^{21,33}$ Using the UPLC method developed here, the MHET and TPA produced by a single degradation reaction can be analysed in under two minutes (Extended Data, Fig. 2). Using our integrated, automated system, over 2000 enzyme variants can be assessed for plastic deconstruction activity in around two days.

The evolutionary strategy comprised of sequential rounds of saturation mutagenesis, using degenerate NNK codons to individually randomise between 24-30 residue positions per cycle. These residues were targeted for mutation based on a number of considerations, including previous reports of their involvement in substrate binding or thermostability, their identification by online protein stability-enhancing tools, or by visual inspection of the protein crystal structure (Extended Data, Fig. 3). Beneficial diversity found during each round was then combined by DNA shuffling. Between rounds 2-3, an additional disulphide bridge (N233C, S282C) was rationally generated in the protein, following reports that the 
inclusion of this structural feature increased protein stability in homologous, promiscuous PET-degrading cutinases. ${ }^{20,34}$

To simultaneously improve both thermostability and activity, the evolutionary pressures applied were gradually increased across rounds by raising both the reaction temperature and extending the reaction time. For rounds 1-4, the primary focus was on improved catalysis at elevated temperatures. Once a satisfactory level of thermostability was achieved, we then added an additional selection pressure of activity on more crystalline material. To this end, the top 3\% of clones identified in rounds 5-6 were screened as purified enzymes against a commercially available semi-crystalline PET powder (cryPET, $36.4 \%$ crystallinity, sourced from Goodfellow) that is more reminiscent of material dominant in post-consumer waste streams. ${ }^{35}$

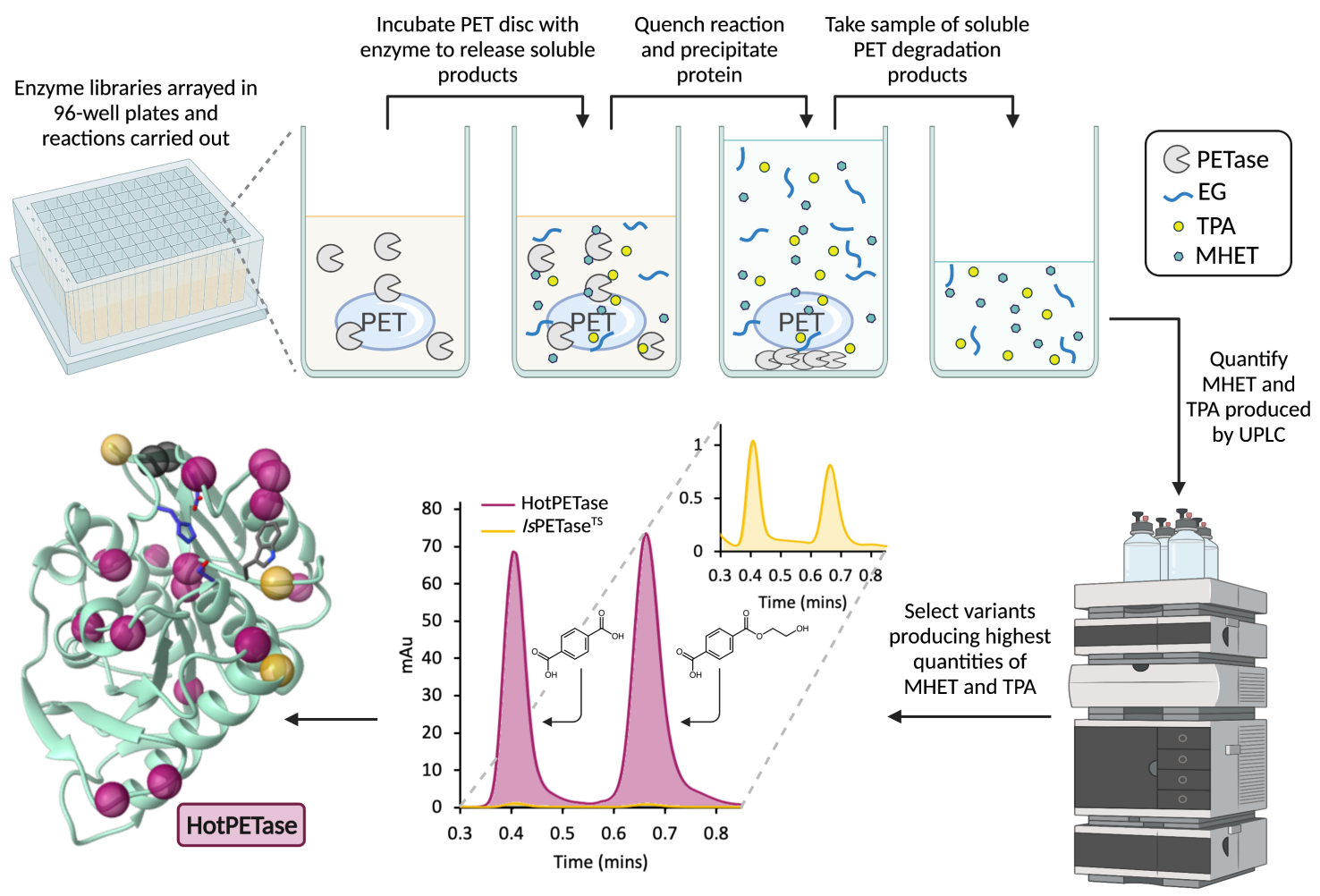

Figure 1. Workflow for the directed evolution of a PETase. Depiction of the laboratory evolution workflow for a single well in a 96-well plate. The example UPLC trace demonstrates a $5 \mathrm{hr}$ PETdegradation reaction (semi-crystalline PET powder, $4 \mathrm{mg} \mathrm{mL}^{-1}$ ) using the best variant following evolution, HotPETase $(0.04 \mu \mathrm{M}$, pink $)$, at $70^{\circ} \mathrm{C}$ as compared to the starting protein IsPETase ${ }^{\mathrm{TS}}$ $(0.04 \mu \mathrm{M}$, yellow). The crystal structure shows the 21 amino acid positions mutated from IsPETase $^{\mathrm{WT}}$ : three positions mutated in the starting protein IsPETase ${ }^{\mathrm{TS}}$ (yellow spheres), 16 installed through evolution (pink spheres) and a rationally installed disulphide bridge (black spheres). The catalytic triad and W185 are shown as blue sticks and grey sticks, respectively. 
The most thermostable and active variant to emerge following six rounds of evolution, HotPETase, contains 21 mutations compared to IsPETase ${ }^{\mathrm{WT}}$ : three from the starting protein template IsPETase ${ }^{\mathrm{TS}}$, two from rational insertion of a disulphide bridge and a further 16 found via directed evolution (Fig. 1). HotPETase has a melting temperature $\left(T_{\mathrm{m}}\right)$ of $82.5^{\circ} \mathrm{C}$, the highest $T_{\mathrm{m}}$ recorded to date of an IsPETase derivative. This elevated thermostability means that the enzyme can be incubated at $80^{\circ} \mathrm{C}$ for 90 minutes with minimal loss in activity (Extended Data, Fig. 4). Variants along the evolutionary trajectory were then compared based on their melting temperatures and their activity in cryPET-deconstruction assays performed at $60^{\circ} \mathrm{C}$ (Extended Data, Fig. 5). As shown in Figure 2a, evolution led to progressive improvements in these two parameters. Whilst both the IsPETase ${ }^{\mathrm{WT}}$ and IsPETase ${ }^{\mathrm{TS}}$ have minimal activity at $60^{\circ} \mathrm{C}$, HotPETase operated well under these conditions.

We next determined the activity of HotPETase across a range of temperatures by monitoring the release of MHET and TPA over time (Fig. 2b). For comparison, analogous experiments were performed using the starting template, IsPETase ${ }^{\mathrm{TS}}$ and the engineered thermostable cutinase LCC ${ }^{\text {ICCG }} .{ }^{20}$ Comparisons of the enzymes were carried out under the optimised buffer conditions for each individual protein, using cryPET powder as the substrate (Extended Data, Fig. 6). At $40^{\circ} \mathrm{C}$, slightly improved initial reaction rates were achieved by HotPETase versus IsPETase $\mathrm{T}^{\mathrm{TS}}$ (Fig. 2b), demonstrating that the evolution of thermostability has not compromised activity at ambient temperatures. While the activity of IsPETase ${ }^{\mathrm{TS}}$ was severely compromised at higher temperatures, the rate of PET-hydrolysis by HotPETase is significantly improved by operating at temperatures approaching the reported $T_{\mathrm{g}}$ of PET in aqueous solutions $\left(\mathrm{ca} .60-65^{\circ} \mathrm{C}\right){ }^{18}$ At $65^{\circ} \mathrm{C}$, each mole of HotPETase releases $2.7 \times 10^{4}$ moles of monomers in one hour, a time-course over which reaction progression is linear. At the same temperature LCC ${ }^{\mathrm{ICCG}}$ produced $5.7 \times 10^{3}$ moles of monomer product in the same time frame, highlighting the superior catalytic activity of this engineered IsPETase. Interestingly, for both HotPETase and $\mathrm{LCC}^{\mathrm{ICCG}}$, the reaction rates were slightly reduced at $70^{\circ} \mathrm{C}$ versus $65^{\circ} \mathrm{C}$.

Comparison of reactions with HotPETase and IsPETase ${ }^{\mathrm{TS}}$ at $40^{\circ} \mathrm{C}$ show that evolution has afforded a more robust catalyst with increased longevity (Fig. 2c, Extended Data, Fig.7). For IsPETase ${ }^{\mathrm{TS}}$, soluble product formation essentially ceases after $8 \mathrm{hrs}$. In contrast, for reactions with HotPETase, monomeric products continue to accumulate for $>48$ hrs. Consistent with previous studies, ${ }^{29}$ the reaction profile is non-linear with faster initial phase for ca. 8 hrs, 
followed by a slower phase from 8-48 hrs. Similar, but more pronounced, non-linear reaction profiles are observed at elevated temperatures (from $60^{\circ} \mathrm{C}-70^{\circ} \mathrm{C}$ ) (Extended Data, Fig. 8). The time course of reactions with HotPETase at $60^{\circ} \mathrm{C}$ demonstrate that product accumulation rises rapidly for the first of $5 \mathrm{hrs}$ of reaction (1.73 $\mathrm{mM}$ of MHET + TPA), but then slows substantially after this time to afford $1.85 \mathrm{mM}$ of product after 48 hrs (Fig. 2d). Product accumulation over time is also non-linear for $\mathrm{LCC}^{\mathrm{ICCG}}$, with 0.57 and $1.50 \mathrm{mM}$ of monomers produced over 5 and 48 hrs respectively, at $60^{\circ} \mathrm{C}$. Addition of fresh HotPETase after $24 \mathrm{hrs}$, leads to similar product accumulation versus time trends as observed at the outset of the reaction (Extended Data, Fig. 9). In contrast, addition of fresh PET substrate does not give rise to any additional soluble products. These observations suggest that reactions stall due to catalyst deactivation, not as a result of inhibition by soluble released products or exhaustion of available plastic substrate. It is interesting to note that during evolution IsPETase libraries were analysed over time-frames ranging from three to seven hours, meaning that limited selection pressure was applied to catalyst longevity at elevated temperatures. We anticipate that adapting selection pressures during future rounds of evolution will lead to improved variants capable of operating efficiently at elevated temperatures for more extended periods.

To improve the extent of PET depolymerisation achievable with HotPETase, we next optimised several reaction parameters including $\mathrm{pH}$, reaction buffer and enzyme loading (Extended Data, Fig. 10-12). Under optimal conditions using $0.5 \mu \mathrm{M}$ HotPETase and cryPET as the substrate $(20 \mathrm{mg}), 6.07 \mathrm{mM}$ of soluble monomer products (MHET:TPA ratio of 1:0.29) were formed within $5 \mathrm{hrs}$, corresponding to degree of depolymerisation of 31\% (Extended Data, Fig. 13). Differential scanning calorimetry (DSC) analysis of samples before and after depolymerisation show an overall increase in crystallinity from $29.8 \%$ to $41.7 \%$, suggesting that HotPETase preferentially degrades the amorphous PET domains (Extended Data, Fig. 14). Interestingly, size-exclusion chromatography (SEC) analysis shows no substantive change in the molecular weight and dispersity of the remaining PET, which may indicate that the enzyme operates in an exo-cleavage fashion, depolymerising individual polymer chains fully before chain transfer to a new macromolecule, thus retaining the original chain lengths in the bulk of the sample. 

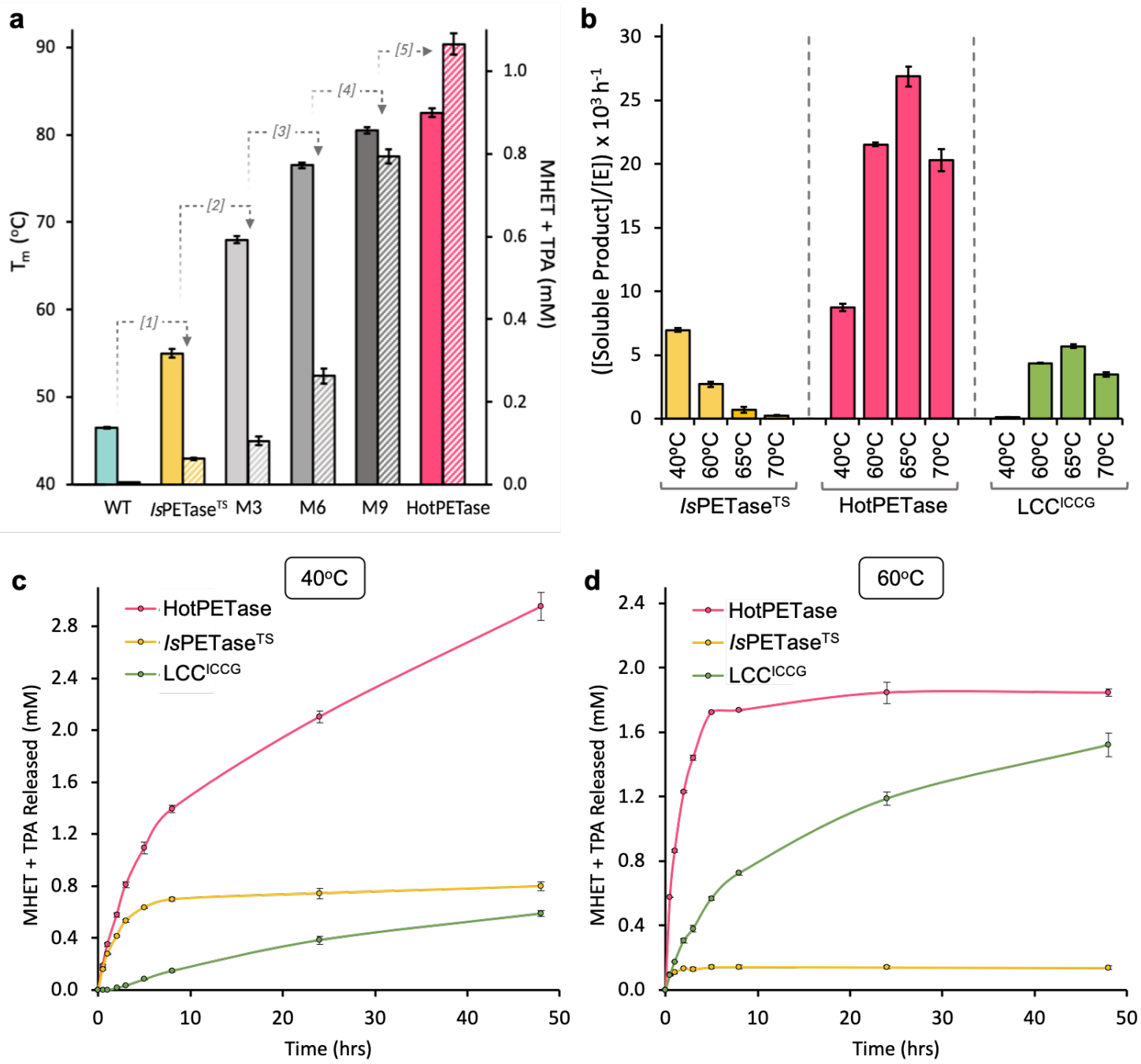

Figure 2. Directed evolution of IsPETase ${ }^{\mathrm{TS}}$ has afforded a more thermostable and active catalyst.

(a) Bar chart demonstrating a snapshot of protein variants along the evolutionary trajectory from IsPETase ${ }^{\mathrm{WT}}$ to HotPETase. Protein variants are on the $\mathrm{x}$-axis; the mutations added to each variant compared to the previous variant are as follows: [1] = S121E, D186H, R280A, [2] = P181V, S207R, S214Y, [3] = Q119K, S213E, N233C, S282C, [4] = R90T, Q182M, N212K, R224L, [5] = S58A, S61V, K95N, M154G, N241C, K252M, T270Q. Solid coloured bars represent the $T_{\mathrm{m}}$ of each variant (left y-axis). Hashed bars represent concentration of MHET and TPA produced by each enzyme variant $(0.04 \mu \mathrm{M})$ over the course of $24 \mathrm{hrs}$, in a reaction at $60^{\circ} \mathrm{C}$ with cryPET $\left(4 \mathrm{mg} \mathrm{mL}^{-1}\right)$ as the substrate. Reaction buffer contained no BugBuster. Reactions were carried out in duplicate; error bars represent the s.d. of the replicates. (b) Bar chart showing enzyme turnovers per hour after one hour of reaction, with cryPET substrate $\left(4 \mathrm{mg} \mathrm{mL}^{-1}\right)$ at different temperatures with IsPETase $^{\mathrm{TS}}\left(0.04 \mu \mathrm{M}\right.$, yellow), HotPETase $\left(0.04 \mu \mathrm{M}\right.$, pink) or LCC ${ }^{\mathrm{ICCG}}(0.04 \mu \mathrm{M}$, green $)$ as the biocatalyst. (c) 48 -hr time-course reactions, with either HotPETase $\left(0.04 \mu \mathrm{M}\right.$, pink), IsPETase ${ }^{\mathrm{TS}}$ $\left(0.04 \mu \mathrm{M}\right.$, yellow), or LCC ${ }^{\mathrm{ICCG}}(0.04 \mu \mathrm{M}$, green) showing total released MHET and TPA in reactions at $40^{\circ} \mathrm{C}$, with cryPET $\left(4 \mathrm{mg} \mathrm{mL}^{-1}\right)$ as the substrate. (d) 48 -hr time-course reactions with HotPETase $\left(0.04 \mu \mathrm{M}\right.$, pink), IsPETase ${ }^{\mathrm{TS}}\left(0.04 \mu \mathrm{M}\right.$, yellow) or $\mathrm{LCC}^{\mathrm{ICCG}}(0.04 \mu \mathrm{M}$, green $)$, showing total released MHET and TPA in reactions at $60^{\circ} \mathrm{C}$, with cryPET $\left(4 \mathrm{mg} \mathrm{mL}^{-1}\right)$ as the substrate. For all reactions, unless specified otherwise, enzymes were assayed at their reported optimal pHs, as detailed in the Methods. Reactions were carried out in triplicate; error bars represent the s.d. of the replicate measurements. 
To further explore the utility of HotPETase, we next attempted to deconstruct commercialgrade PET materials. HotPETase can depolymerise milled bottle-grade PET (41.9\% crystallinity), albeit with a reduced conversion compared to that observed with cryPET powder (Fig. 3). To showcase the selectivity achievable with biocatalytic depolymerisations, HotPETase was used to deconstruct a common laminated packaging film comprised of PET and polyethylene (PE). Importantly, this PET/PE laminate is challenging to recycle mechanically, and indeed is considered a pollutant in commercial recycling streams. The HotPETase enzyme is adept at selectively deconstructing the PET portion of this material, releasing $9.2 \mathrm{mM}$ of soluble PET-derived monomers over the course of $24 \mathrm{hrs}$ (Fig. 3a). Interestingly the degree of depolymerisation of the PET/PE laminate, which has an initial crystallinity of $1.5 \%$, is higher than that achieved with semi-crystalline cryPET powder and milled bottle-grade PET. Scanning electron microscopy (SEM) reveals significant pitting of the PET surface, whereas the PE surface appears unchanged, compared to control reactions run in the absence of enzyme (Fig. 3b, Extended Data, Fig.16).

a

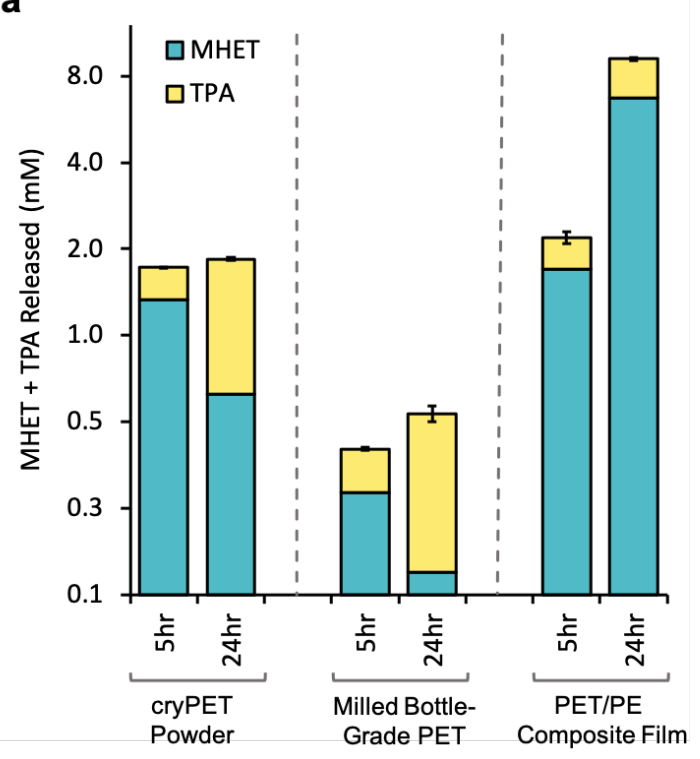

b

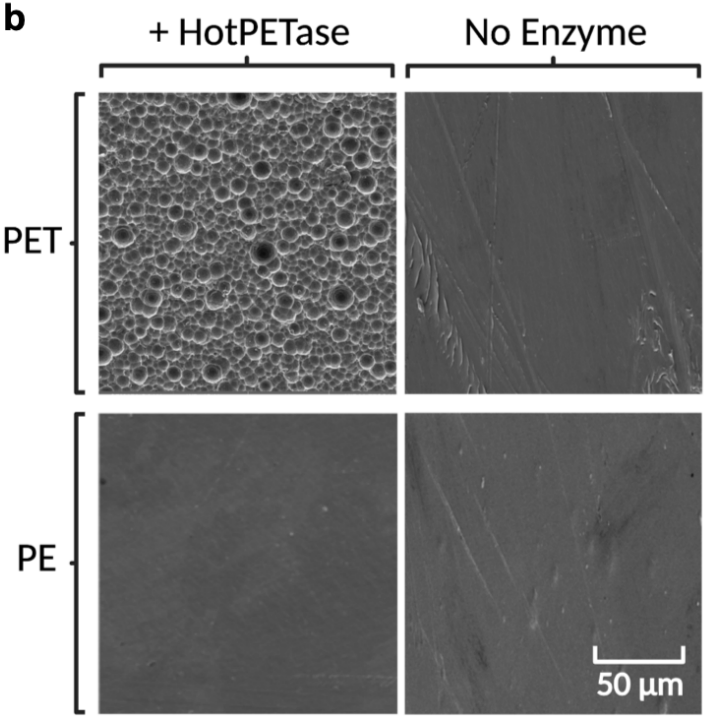

Figure. 3. Biocatalytic deconstruction of a range of PET-based materials by HotPETase. (a) Bar chart showing soluble aromatic PET degradation products MHET (blue) and TPA (yellow) produced following reaction of HotPETase $(0.04 \mu \mathrm{M})$ at $60^{\circ} \mathrm{C}$ over the course of $24 \mathrm{hrs}$ with each PET substrate $\left(4 \mathrm{mg} \mathrm{mL}^{-1}\right)$. HotPETase has activity on crystalline PET powder (cryPET), milled bottle grade PET (bgPET) and a PET/PE composite packaging film lid. Reactions were carried out in triplicate; error bars represent the s.d. of the total MHET and TPA produced in the replicates. The y-axis is on a logarithmic scale. Physical characterisation of the substrates preand post-enzymatic deconstruction is detailed in Extended Data, Table 4 and Extended Data, Fig. 15. (b) SEM images of both sides of a PET/PE packaging material degraded in the presence or absence of HotPETase, in a reaction at $40^{\circ} \mathrm{C}$ over the course of six days. SEM images for reactions at $60^{\circ} \mathrm{C}$ are provided in Extended Data, Fig. 16. 
To gain insights into the origins of HotPETase thermostability and its improved activity, the crystal structure of the enzyme was solved and refined to a resolution of $2.2 \AA$ for comparison to the starting variant IsPETase ${ }^{\mathrm{TS}}$. The structures of HotPETase and IsPETase ${ }^{\mathrm{TS}}$ superimpose well, with a root-mean-square-deviation of $1.18 \AA$ (Fig. 4a). In HotPETase, the disulphide bridge between the newly introduced Cys233 and Cys282 pair is formed as intended, with an S-S interatomic distance of $2.03 \AA$ (Fig. 4b). The P181V mutation results in an additional hydrogen bond between Vall81 and Leul99 leading to better packing of the central $\beta$-sheet region compared to IsPETase ${ }^{\mathrm{TS}}$, which is now more reminiscent of thermostable cutinases (Fig. 4c). Analysis of the surface charge distributions of HotPETase and IsPETase ${ }^{\mathrm{TS}}$ reveals substantial changes in the putative polymer binding cleft to reduce positive charge in the evolved enzyme (Extended Data, Fig. 17). Further computational analysis and structural studies with bound substrate analogues is needed to correlate the observed changes in surface electrostatics to polymers positioning and binding affinity. Interestingly the 'wobbling' tryptophan, Trp185, a feature which is thought to aid substrate binding and catalysis in the wildtype enzyme, ${ }^{23,36}$ is present as a single well-defined conformer in the HotPETase structure. Extensive remodelling of the loop region connecting $\beta 7-\alpha 5$, including introduction of a bulky Tyr214, leads to a new $\pi$-stacking interaction with Trp185 that restricts its conformational freedom (Fig. 4d). To explore the functional significance of the altered environment around Trp185, mutated residues installed in the $\beta 7$ $\alpha 5$ connecting loop were reverted back to their wildtype amino acids (HotPETase ${ }^{\mathrm{LR}}(\mathrm{K} 212 \mathrm{~N}$, E213S, Y214S, C215N)). These modifications led to a substantial $7.5^{\circ} \mathrm{C}$ reduction in $T_{\mathrm{m}}$ and compromised catalytic performance at elevated temperatures (Extended Data, Fig. 18). Interestingly, catalytic activity at low temperatures is minimally affected, suggesting that in the heavily engineered HotPETase, the fixed conformation of Trp185 is not detrimental to catalysis. Combined these results suggest that a flexible Trp185 is not a pre-requisite for efficient PET deconstruction. 


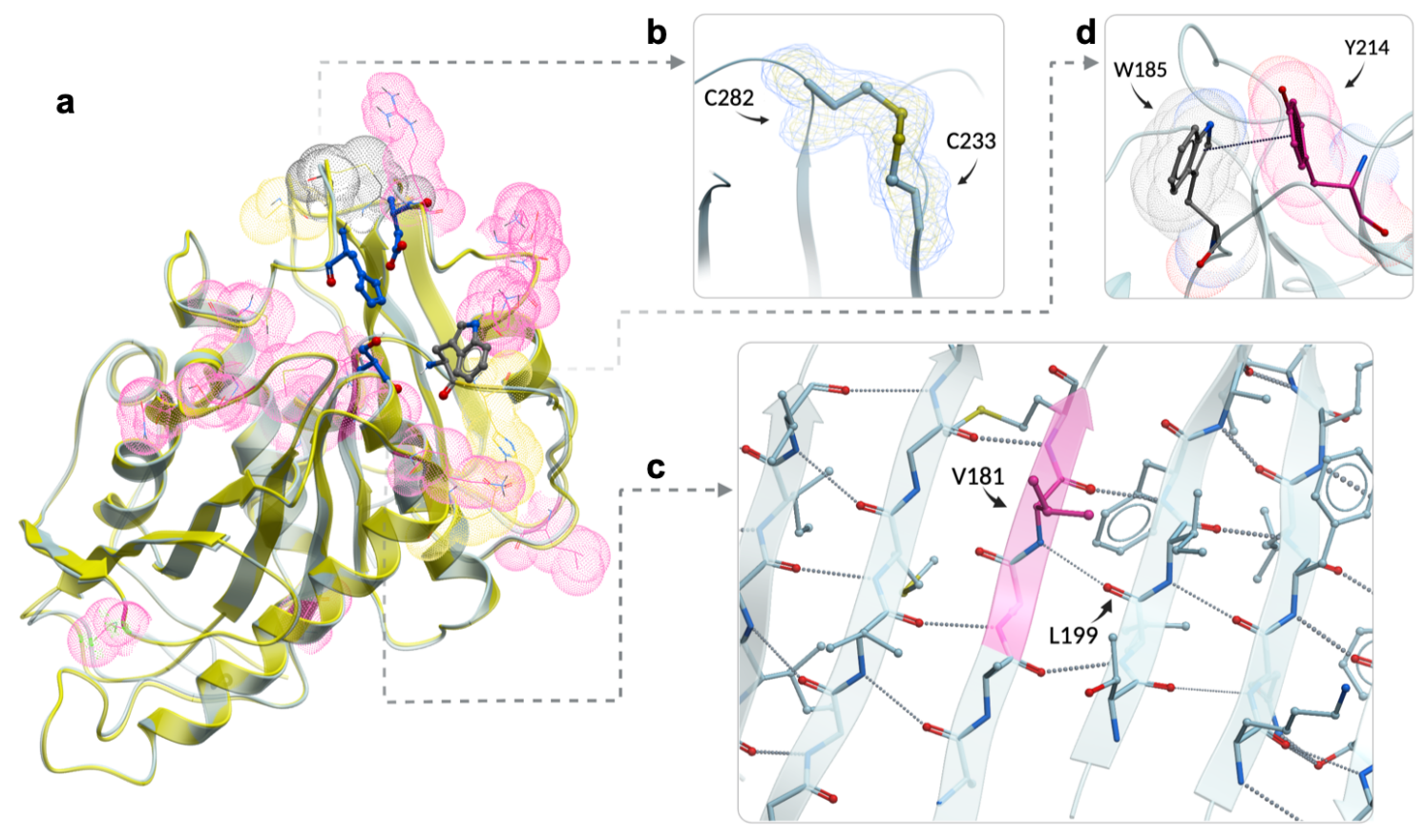

Figure 4. Comparison of crystal structures and features of HotPETase and IsPETase ${ }^{\mathrm{TS}}$. (a) A global superposition of IsPETase ${ }^{\mathrm{TS}}$ (yellow) and HotPETase (light blue). Mutations in IsPETase $^{\text {TS }}$ compared to IsPETase ${ }^{\mathrm{WT}}$ are highlighted with yellow spheres. Mutations installed by directed evolution are highlighted with pink spheres. The rationally inserted disulphide bridge is highlighted with black spheres. The catalytic triad and W185 are represented as blue and grey sticks, respectively. (b) The disulphide bridge is correctly formed between C233 and C282 in HotPETase. Electron density is $2 \mathrm{Fo}$-Fc contoured at 1 sigma (blue) and 2 sigma (yellow). (c) The conversion of P181 in IsPETase ${ }^{\mathrm{TS}}$ to V181 in HotPETase (highlighted pink) results in extension of $\beta$-sheet 6, and the formation of an additional hydrogen bond (dashed lines) to L199.

\section{Discussion}

Directed evolution has proven to be the most versatile and comprehensive strategy for engineering industrial biocatalysts. Somewhat surprisingly, despite great interest in enzymatic plastic deconstruction, PETase engineering efforts to date have focused on structure guided design using computational methods. The notable absence of PETases engineered via laboratory evolution likely reflects the challenges of developing suitable high-throughput, quantitative methods for analysing the catalytic deconstruction of insoluble polymers. Here, we have developed an automated directed evolution platform for engineering plastic deconstructing enzymes and showcase its utility, through the development of an evolved thermostable PETase (HotPETase, $T_{\mathrm{m}}=82.5^{\circ} \mathrm{C}$ ) that can operate at the glass transition temperature of PET and depolymerises semi-crystalline PET more rapidly than previously reported PETases. Importantly, HotPETase is able to deconstruct 
commercial bottle-grade PET and can selectively deconstruct PET in a PET/PE laminated packaging material, highlighting the potential benefits of enzymatic depolymerisations for real-world samples with minimal pre-treatment or processing. Structural characterisation of HotPETase highlights formation of a new disulphide bridge and improved packing of the central $\beta$-sheet region which likely aid thermostability, alongside the presence of a single well-defined conformer of Trp185, indicating that flexibility of this tryptophan is not a prerequisite for effective catalysis. To maximise the utility of our platform moving forward, it will be important to interface our evolution methods with alternative strategies for augmenting biocatalyst function, including computationally guided engineering, ${ }^{37}$ introduction of polymer binding domains, ${ }^{38}$ and the development of multi-enzyme complexes. $^{39}$ Likewise, combining and optimising biocatalytic deconstructions with enzymatic monomer upcycling methods will be an important avenue for exploration. ${ }^{14,40}$

In the future, we anticipate that by adapting the selection pressures of our directed evolution workflows, we will be able to engineer a suite of useful biocatalysts with complementary functions and improved activities under process-relevant conditions. For example, we can extend catalyst stability and lifetime by increasing reaction times and temperatures, optimise biocatalysts to act upon alternative plastic substrates, or enhance enzyme specificities in order that they operate on single polymer components from mixed plastic waste streams. In doing so, our laboratory evolution platform will contribute to a biocatalytic recycling strategy to recover value from plastic waste.

\section{Acknowledgements}

We acknowledge generous support from the Biotechnology and Biological Sciences Research Council (David Phillips Fellowship BB/M027023/1 to A.P.G), the European Research Council (ERC Starter Grant, no. 757991 to A.P.G), the Medical Research Council and Buddi (MRC PhD funding to E.L.B), the UK Catalysis Hub (funded through EP/R026815/1, EP/K014706/2, EP/K014668/1, EP/K014854/1, EP/K014714/1, and EP/M013219/1), the EPSRC (EP/R031711/1 to SJH and SR) and the Henry Royce Institute for Advanced Materials (funded through EP/R00661X/1, EP/S019367/1, EP/P025021/1 and EP/P025498/1). We are grateful to Diamond Light Source for time on beamlines i03, i04 and i04-1 under proposals MX12788-50, MX17773-25, MX17773-34 and MX17773-52, and to Manchester SYNBIOCHEM Centre (BB/M017702/1) and the Future Biomanufacturing Hub (EP/SO1778X/1) for access to their facilities. 


\section{References}

1. PlasticsEurope. Plastics- the Facts 2019, An analysis of European plastics production, demand and waste data. (2019).

2. Borrelle, S. et al. Predicted growth in plastic waste exceeds efforts to mitigate plastic pollution. Science 369, (2020).

3. Burgess, M., Holmes, H., Sharmina, M. \& Shaver, M. P. The future of UK plastics recycling: One Bin to Rule Them All. Resources, Conservation and Recycling 164, 105191 (2021).

4. Geyer, R., Jambeck, J. \& Law, K. Production, use, and fate of all plastics ever made. Science Advances 3, el700782 (2017).

5. Jambeck, J. R. et al. Plastic waste inputs from land into the ocean. Science 347, 768-771 (2015).

6. Laville, S. \& Taylor, M. A million bottles a minute: world's plastic binge 'as dangerous as climate change".' The Guardian 28, (2017).

7. Ellen MacArthur Foundation. The New Plastics Economy: Rethinking the future of plastics $\mathcal{E}$ catalysing action. https://www.ellenmacarthurfoundation.org/publications/the-newplastics-economy-rethinking-the-future-of-plastics-catalysing-action (2017).

8. Rahimi, A. \& García, J. M. Chemical recycling of waste plastics for new materials production. Nature Reviews Chemistry 1, 0046 (2017).

9. Jehanno, C. et al. Organocatalysed depolymerisation of PET in a fully sustainable cycle using thermally stable protic ionic salt. Green Chemistry 20, 1205-1212 (2018).

10. Karayannidis, G., Chatziavgoustis, A. P. \& Achilias, D. Poly(ethylene terephthalate) recycling and recovery of pure terephthalic acid by alkaline hydrolysis. Advances in Polymer Technology 21, 250-259 (2002).

11. Korley, L., Epps, T., Helms, B. \& Ryan, A. Toward polymer upcycling-adding value and tackling circularity. Science 373, 66-69 (2021).

12. Zhu, B., Wang, D. \& Wei, N. Enzyme Discovery and Engineering for Sustainable Plastic Recycling. Trends in Biotechnology (2021) doi:10.1016/j.tibtech.2021.02.008.

13. Zimmermann, W. Biocatalytic recycling of polyethylene terephthalate plastic. Philosophical Transactions of the Royal Society A: Mathematical, Physical and Engineering Sciences 378, 20190273 (2020).

14. Sadler, J. C. \& Wallace, S. Microbial synthesis of vanillin from waste poly(ethylene terephthalate). Green Chemistry 23, 4665-4672 (2021).

15. Andrady, A. L. Assessment of Environmental Biodegradation of Synthetic Polymers. Journal of Macromolecular Science, Part C 34, 25-76 (1994).

16. Herrero Acero, E. et al. Enzymatic Surface Hydrolysis of PET: Effect of Structural Diversity on Kinetic Properties of Cutinases from Thermobifida. Macromolecules 44, 4632-4640 (2011).

17. Sulaiman, S. et al. Isolation of a novel cutinase homolog with polyethylene terephthalatedegrading activity from leaf-branch compost by using a metagenomic approach. Applied and Environmental Microbiology 78, 1556-62 (2012).

18. Kawai, F. et al. A novel $\mathrm{Ca}^{2+}$-activated, thermostabilized polyesterase capable of hydrolyzing polyethylene terephthalate from Saccharomonospora viridis AHK190. Applied Microbiology and Biotechnology 98, 10053-10064 (2014).

19. Ribitsch, D. et al. Hydrolysis of polyethylene terephthalate by $p$-nitrobenzylesterase from Bacillus subtilis. Biotechnology Progress 27, 951-960 (2011). 
20. Tournier, V. et al. An engineered PET depolymerase to break down and recycle plastic bottles. Nature 580, 216-219 (2020).

21. Yoshida, S. et al. A bacterium that degrades and assimilates poly(ethylene terephthalate). Science 351, 1196-9 (2016).

22. Austin, H. P. et al. Characterization and engineering of a plastic-degrading aromatic polyesterase. Proceedings of the National Academy of Sciences of the United States of America 115, E4350-E4357 (2018).

23. Han, X. et al. Structural insight into catalytic mechanism of PET hydrolase. Nature Communications 8, 2106 (2017).

24. Joo, S. et al. Structural insight into molecular mechanism of poly(ethylene terephthalate) degradation. Nature Communications 9, 382 (2018).

25. Ronkvist, M. A. sa, Xie, W., Lu, W. \& Gross, R. A. Cutinase-Catalyzed Hydrolysis of Poly(ethylene terephthalate). Macromolecules 42, 5128-5138 (2009).

26. Wei, R. \& Zimmermann, W. Biocatalysis as a green route for recycling the recalcitrant plastic polyethylene terephthalate. Microbial Biotechnology 10, 1302-1307 (2017).

27. Son, H. F. et al. Rational Protein Engineering of Thermo-Stable PETase from Ideonella sakaiensis for Highly Efficient PET Degradation. ACS Catalysis 9, 3519-3526 (2019).

28. Son, H. F. et al. Structural bioinformatics-based protein engineering of thermo-stable PETase from Ideonella sakaiensis. Enzyme and Microbial Technology 141, 109656 (2020).

29. Cui, Y. et al. Computational Redesign of a PETase for Plastic Biodegradation under Ambient Condition by the GRAPE Strategy. ACS Catalysis 11, 1340-1350 (2021).

30. Ma, Y. et al. Enhanced Poly(ethylene terephthalate) Hydrolase Activity by Protein Engineering. Engineering 4, 888-893 (2018).

31. Arnold, F. H. Directed Evolution: Bringing New Chemistry to Life. Angewandte Chemie International Edition 57, 4143-4148 (2018).

32. Zeymer, C. \& Hilvert, D. Directed Evolution of Protein Catalysts. Annual Review of Biochemistry 87, 131-57 (2018).

33. Zhong-Johnson, E., Voigt, C. \& Sinskey, A. An absorbance method for analysis of enzymatic degradation kinetics of poly(ethylene terephthalate) films. Scientific Reports 11, (2021).

34. Then, J. et al. A disulfide bridge in the calcium binding site of a polyester hydrolase increases its thermal stability and activity against polyethylene terephthalate. FEBS Open Bio 6, 425-432 (2016).

35. Ügdüler, S. et al. Towards closed-loop recycling of multilayer and coloured PET plastic waste by alkaline hydrolysis. Green Chemistry 22, 5376-5394 (2020).

36. Chen, C.-C. et al. General features to enhance enzymatic activity of poly(ethylene terephthalate) hydrolysis. Nature Catalysis 4, 425-430 (2021).

37. Huang, P.-S., Boyken, S. E. \& Baker, D. The coming of age of de novo protein design. Nature 537, 320 (2016).

38. Dai, L. et al. Enhancing PET hydrolytic enzyme activity by fusion of the cellulose-binding domain of cellobiohydrolase I from Trichoderma reesei. Journal of Biotechnology 334, 4750 (2021).

39. Knott, B. C. et al. Characterization and engineering of a two-enzyme system for plastics depolymerization. Proceedings of the National Academy of Sciences 117, 25476 (2020).

40. Rorrer, N. A. et al. Combining Reclaimed PET with Bio-based Monomers Enables Plastics Upcycling. Joule 3, 1006-1027 (2019). 